\title{
ON PARALLEL DISPLACEMENT IN A NON-FINSLER SPACE*†
}

\author{
BY \\ HOMER VINCENT CRAIG
}

\section{INTRODUCTION}

Preliminary definitions and concepts. $\ddagger$ In modern geometry any set of objects in a one-to-one reciprocal correspondence with the totality of sets of ordered numbers $x \equiv\left(x^{1}, x^{2}, \cdots, x^{n}\right)$ is called an $n$-dimensional space; and any particular one-to-one correspondence between the objects and a set $x$ is called a coördinate system. If in addition to a space there is given a curvilinear integral, the "arc length," the space is said to be metric. Thus any space can be regarded as the bearer of an indefinite number of metric spaces.

A set of $n$ equations

$$
y^{i}=y^{i}\left(x^{1}, x^{2}, \cdots, x^{n}\right)
$$

such that the functions $y^{i}$ are single-valued for all points $x$ of our $n$-dimensional space, and which can be solved so as to yield a set of $n$ equations

$$
x^{\alpha}=x^{\alpha}\left(y^{1}, y^{2}, \cdots, y^{n}\right)
$$

in which the functions $x^{\alpha}$ are single-valued, determines a one-to-one correspondence between the sets of numbers $x$ and $y$. Such a set of equations is said to define a transformation of coördinates.

An object of any sort which is not changed by transformations of coördinates is called an invariant. For example any point is an invariant, and likewise any point function. In this paper we require that the functions which define the transformations involved be analytic and have a nonvanishing Jacobian. Invariants of this restricted group of transformations are known as differential invariants. An invariant may consist of a single function or of a number of functions. In the latter case the individual functions are called the components of the invariant. An invariant is said to be a tensor if its components transform according to equations of the type

\footnotetext{
* Presented to the Society, June 20, 1929; received by the editors May 8, 1930.

† This paper is a part of a thesis written at the University of Wisconsin under the direction of Professor J. H. Taylor.

$\ddagger$ This material is taken from Oswald Veblen, The Invariants of Quadratic Differential Forms, Cambridge, 1927.
} 


$$
\bar{T}_{j_{1} j_{2} \cdots j_{m}}^{i_{1} i_{2} \cdots i_{q}}=\left|\frac{\partial x}{\partial y}\right|^{n} \sum_{\alpha, \beta}^{1, n} T_{\beta_{1} \beta_{2} \cdots \beta_{m}}^{\alpha_{1} \alpha_{2} \cdots \alpha_{q}} \frac{\partial y^{i_{1}}}{\partial x^{\alpha_{1}}} \cdots \frac{\partial y^{i_{q}}}{\partial x^{\alpha_{q}}} \frac{\partial x^{\beta_{1}}}{\partial y^{j_{1}}} \cdots \frac{\partial x^{\beta_{m}}}{\partial y^{j_{m}}} .
$$

The number $n$ is called the weight of the tensor, $m$ its order of covariance, and $q$ its order of contravariance.

Notation. Throughout this paper we shall employ letters without indices to represent sets; and shall use primes to indicate differentiation with respect to the parameter which is used to define the curve in question. Thus $x, x^{\prime}, x^{\prime \prime}, x^{\prime \prime \prime}$ shall designate respectively the sets

$$
x^{i}, \frac{d x^{i}}{d t}, \frac{d^{2} x^{i}}{d t^{2}}, \frac{d^{3} x^{i}}{d t^{3}} \quad(i=1,2, \cdots, n) .
$$

Partial derivatives will be denoted by means of subscripts; thus

$$
F_{x^{\prime}} \alpha\left(x, x^{\prime}, x^{\prime \prime}\right) \equiv \frac{\partial F\left(x, x^{\prime}, x^{\prime \prime}\right)}{\partial x^{\prime \alpha}} .
$$

In the case of the functions $F, f$, and $H$ to be introduced below, this notation will be modified by omitting the $x$ from the subscript when the differentiation is with respect to the highest derivatives present, i.e.

$$
F_{\alpha}\left(x, x^{\prime}, x^{\prime \prime}\right)=\frac{\partial F\left(x, x^{\prime}, x^{\prime \prime}\right)}{\partial x^{\prime \prime \alpha}} .
$$

(We shall prove later that the quantities $F_{\alpha \beta \cdots \lambda}$ are the components of a covariant tensor.) Finally, summations are to be understood, as in tensor analysis, when repeated indices occur. Thus, for example, $x^{\prime \alpha} F_{\alpha}$ shall designate the sum

$$
x^{\prime 1} F_{1}+x^{\prime 2} F_{2}+\cdots+x^{\prime n} F_{n} .
$$

Riemannian geometry. If the metric of the space is given by the integral of the square root of a quadratic differential form,

$$
g_{\alpha \beta} x^{\prime \alpha} x^{\prime \beta} \text {, }
$$

the space is said to be Riemannian; and the theory of such forms is known as Riemannian geometry. The coefficients $g_{\alpha \beta}$ are the components of a covariant tensor, while the quantities $g^{\alpha \beta}$ (the matrix $\left(g^{\alpha \beta}\right)$ is the inverse of the matrix $\left.\left(g_{\alpha \beta}\right)\right)$ are the components of a contravariant tensor. These two tensors are called, respectively, the fundamental covariant tensor, and the fundamental contravariant tensor. An invariant of great importance $[\alpha \beta, \gamma]$ is derived from $g_{\alpha \beta}$ in accordance with the formula

$$
[\alpha \beta, \gamma] \equiv \frac{1}{2}\left[\frac{\partial g_{\alpha \gamma}}{\partial x^{\beta}}+\frac{\partial g_{\beta \gamma}}{\partial x^{\alpha}}-\frac{\partial g_{\alpha \beta}}{\partial x^{\gamma}}\right]
$$


The law of transformation of $[\alpha \beta, \gamma]$ is expressed by the equation

$$
[\overline{i j, k}]=[\alpha \beta, \gamma] \frac{\partial x^{\alpha}}{\partial y^{i}} \frac{\partial x^{\beta}}{\partial y^{j}} \frac{\partial x^{\gamma}}{\partial y^{k}}+g_{\alpha \beta} \frac{\partial x^{\alpha}}{\partial y^{k}} \frac{\partial^{2} x^{\beta}}{\partial y^{i} \partial y^{j}} .
$$

On multiplying the two members of this relation by the corresponding members of the equality

$$
\bar{g}^{l k} \frac{\partial x^{\delta}}{\partial y^{l}}=g^{\delta \gamma} \frac{\partial y^{k}}{\partial x^{\gamma}}
$$

which expresses the contravariance of $g^{\delta \gamma}$, we obtain

$$
\bar{g}^{l k}[\overline{i j, k}] \frac{\partial x^{\delta}}{\partial y^{l}}=g^{\delta \gamma}[\alpha \beta, \gamma] \frac{\partial x^{\alpha}}{\partial y^{i}} \frac{\partial x^{\beta}}{\partial y^{j}}+\frac{\partial^{2} x^{\delta}}{\partial y^{i} \partial y^{j}} .
$$

This last relationship is known as the fundamental affine connection, while the quantities $[\alpha \beta, \gamma]$ and

$$
g^{\delta \gamma}[\alpha \beta, \gamma] \equiv\left\{\alpha_{\beta}^{\delta}\right\}
$$

are called, respectively, the Christoffel symbols of the first and second kind.

The fundamental affine connection enables one to derive tensors from tensors by differentiation. Consider, for example, a vector $V$ defined along a given curve by means of the parameter $t$. Let us differentiate the equation for the transformation of $V$, i.e., $V^{\delta}=\bar{V}^{i} \partial x^{\delta} / \partial y^{i}$. We obtain thereby

$$
V^{\prime \delta}=\overline{V^{\prime}} \frac{\partial x^{\delta}}{\partial y^{i}}+\bar{V}^{i} \frac{\partial^{2} x^{\delta}}{\partial y^{i} \partial y^{i}} y^{\prime i},
$$

and in this, the second derivatives may be eliminated by means of the fundamental affine connection; thus

$$
V^{\prime \delta}=\bar{V}^{\prime i} \frac{\partial x^{\delta}}{\partial y^{i}}+\bar{V}^{i} y^{\prime} i\left[\left\{\begin{array}{c}
l \\
i j
\end{array}\right\} \frac{\partial x^{\delta}}{\partial y^{l}}-\left\{\begin{array}{c}
\delta \\
\alpha \beta
\end{array}\right\} \frac{\partial x^{\alpha}}{\partial y^{i}} \frac{\partial x^{\beta}}{\partial y^{i}}\right] .
$$

Transposing the final term, which may be written in the form $V^{\alpha} x^{\prime \beta}\left\{\alpha_{\beta}^{\delta}\right\}$, we note that

$$
V^{\prime \delta}+V^{\alpha} x^{\prime \beta}\left\{\begin{array}{c}
\delta \\
\alpha \beta
\end{array}\right\}
$$

is a contravariant vector. If the vector derived from $V$ by this differentiation process is zero, then it is said that $V$ "undergoes parallel displacement" or that $V$ "remains parallel." If the tangent vector of a curve satisfies this definition of parallel displacement, then the curve is said to be autoparallel. 
It is a theorem of Riemannian geometry that the magnitude of a vector, and the cosine of the angle between vectors, remain unaltered when the vectors undergo parallel displacement. The invariants magnitude of a vector and cosine of the angle between vectors are defined, respectively by the following formulas:

$$
\left(g_{\alpha \beta} \xi^{\alpha} \xi^{\beta}\right)^{1 / 2} ; \frac{g_{\alpha \beta} \xi^{\alpha} \eta^{\beta}}{\left(g_{\alpha \beta} \xi^{\alpha} \xi^{\beta}\right)^{1 / 2}\left(g_{\alpha \beta} \eta^{\alpha} \eta^{\beta}\right)^{1 / 2}} .
$$

The geometry of paths. * One generalization of Riemannian geometry has been obtained by replacing the quantities $\left\{\alpha_{\alpha}{ }_{\beta}\right\}$ in the affine connection by a set of point functions $\Gamma_{\alpha \beta}^{\gamma}$. The problem of finding a Riemannian metric such that

$$
\left\{\begin{array}{c}
\gamma \\
\alpha \beta
\end{array}\right\}=\Gamma_{\alpha \beta}^{\gamma}
$$

does not always admit of a solution, and so Riemannian geometry is regarded as a special case of the geometry of paths. The system of curves defined by the set of differential equations

$$
x^{\prime \prime i}+\Gamma_{j k}^{i} x^{\prime j} x^{\prime k}=0 \quad(i=1,2, \cdots, n)
$$

are regarded as autoparallel and are called the paths.

Finsler geometry. A second generalization of Riemannian geometry due to Emmy Noether, $\dagger$ Finsler, $\ddagger$ and Berwald, $\S$ may be obtained by replacing $\left(g_{\alpha \beta} x^{\prime \alpha} x^{\prime \beta}\right)^{1 / 2}$ by a more general function $F\left(x, x^{\prime}\right)$ of $x$ and $x^{\prime}$. The restrictions on $F$ are for the most part those needed to insure the regularity of the problem of minimizing the integral $\int F\left(x, x^{\prime}\right) d t$. A fundamental tensor is derived by differentiating $\frac{1}{2} F^{2}$ with respect to $x^{\prime \alpha}$ and $x^{\prime \beta}$, and the parameter is so selected that $F$ maintains the value one along the path of integration. We point out that the $g_{\alpha \beta}$ of Riemannian geometry may be obtained in this way from $\left(g_{\alpha \beta} x^{\prime \alpha} x^{\prime \beta}\right)^{1 / 2}$ and that as a consequence of the choice of parameter $\int F^{2} d t=\int F d t$. The covariant tensor $F_{\alpha \beta}$ cannot be used as a fundamental tensor since its determinant vanishes (due to the conditions imposed on $F$ ). The invariants

$$
\left(f_{\alpha \beta} \xi^{\alpha} \xi^{\beta}\right)^{1 / 2} ; \frac{f_{\alpha \beta} \xi^{\alpha} \eta^{\beta}}{\left(f_{\alpha \beta} \xi^{\alpha} \xi^{\beta}\right)^{1 / 2}\left(f_{\alpha \beta} \eta^{\alpha} \eta^{\beta}\right)^{1 / 2}} \quad\left(f=\frac{1}{2} F^{2}\right)
$$

* See Oswald Veblen and L. P. Eisenhart, The Riemannian geometry and its generalization, Proceedings of the National Academy of Sciences, vol. 8 (1922), p. 19.

$\dagger$ Emmy Noether, Invarianten beliebiger Differentialausdrucke, Göttinger Nachrichten, Mathematisch-Physikalische Klasse, 1918, pp. 37-44.

$\ddagger$ P. Finsler, Über Kurven und Flächen in allgemeinen Räumen, Dissertation, Göttingen, 1918.

$\S \mathrm{L}$. Berwald, Über Parallelïbertragung in Räumen mit allgemeiner Massbestimmung, Jahrsbericht der Deutschen Mathematiker Vereinigung, vol. 34 (1925). 
are called, respectively, the magnitude of $\xi$, and the cosine of the angle between $\xi$ and $\eta$, because of their analogy to the like named Riemannian invariants. J. H. Taylor* and independently J. L. Synge have constructed a differentiation process analogous to the one described in the section on Riemannian geometry. Their process gives rise to a theory of parallelism which has the cardinal properties that the magnitude, and the "direction" of vectors undergoing parallel displacement are invariant.

Purpose of this paper. The principle object of this paper is to develop a differentiation process and a theory of parallelism for a space whose metric is given by a function $F\left(x, x^{\prime}, x^{\prime \prime}\right)$ involving $x^{\prime \prime}$ as well as $x^{\prime}$ and $x$.

The metric. We shall require that (a), $F\left(x, x^{\prime}, x^{\prime \prime}\right)$ be of class $\dagger$ three, (b), the classical " $F$ one" function $\ddagger$ associated with the problem of minimizing the integral

$$
J=\int_{t_{1}}^{t_{2}} F\left(x, x^{\prime}, x^{\prime \prime}\right) d t
$$

be different from zero, (c), $J$ be independent of the choice of parameter. Specifically, (a) and (b) are to hold not merely along a certain curve but throughout the region under consideration and for all values of the sets $x^{\prime}, x^{\prime \prime}$ excepting $x^{\prime 1}=0 ; x^{\prime 2}=0 ; \cdots ; x^{n}=0$; which values shall be excluded; (c) is to hold along all regular curves lying in the region for all choices of $t_{1}$ and $t_{2}$.

It is worthy of note that this invariance of $J$ is equivalent to the condition that

$$
F\left(x, x^{\prime}, x^{\prime \prime}\right) \equiv I\left(x, x^{\prime}, x^{\prime \prime}\right) H\left(x, x^{\prime}\right),
$$

where $I$ is a function that is invariant in functional form under a change of parameter and $H$ is homogeneous of degree plus one in $x^{\prime}$. Since the invariance is supposed to hold for all values of $t_{1}$ and $t_{2}$ within certain limits, it is equivalent to the invariance of $F\left(x, x^{\prime}, x^{\prime \prime}\right) d t, \S$ that is,

where

$$
F\left(x, x^{\prime}, x^{\prime \prime}\right) d t=F\left(X(\tau), \frac{d X(\tau)}{d \tau}, \frac{d^{2} X(\tau)}{d \tau^{2}}\right) d \tau
$$

$$
t=t(\tau), \tau=\tau(t) ; \quad d t=\frac{d t(\tau)}{d \tau} d \tau
$$

* J. H. Taylor, A generalization of Levi-Civita's parallelism and the Frenet formulas, these Transactions, vol. 27 (1925).

† Oscar Bolza, Vorlesungen über Variationsrechnung, 1909, p. 13.

$\ddagger$ Oscar Bolza, loc. cit., p. 196.

\& See Oscar Bolza, loc. cit., p. 193. 
is the parameter transformation, $t(\tau)$ being an increasing function of class $C^{\prime \prime}$, and $X(\tau)=x(t(\tau))$. Evidently $F$ will have the desired invariance property if it is of the above form, and conversely, if $F\left(x, x^{\prime}, x^{\prime \prime}\right) d t$ is invariant, then

$$
F\left(x, x^{\prime}, x^{\prime \prime}\right) / H\left(x, x^{\prime}\right), \quad H \neq 0, \text { and homogeneous, }
$$

is invariant in functional form under a parameter transformation and $F=(F / H) H$. Because of this fact we shall adopt the view that our space was generated from a Finsler space $H\left(x, x^{\prime}\right)$ by a "warping" such that the length of each curve element $H d t$ of the original space is changed to $I H d t$. Accordingly, we shall consider that the metric properties of $F$ depend upon the Finsler space from which $F$ originated.

Zermelo has shown that the independence of $J$ upon the parameter implies the following identities in $x, x^{\prime}, x^{\prime \prime}$ :

$$
\begin{aligned}
x^{\prime \alpha} F_{\alpha} & \equiv 0, \\
x^{\prime \alpha} F_{x^{\prime} \alpha}+2 x^{\prime \prime \alpha} F_{\alpha} & \equiv F \quad \text { (the range of } \alpha \text { is } 1 \text { to } n \text { ). }
\end{aligned}
$$

Evidently, (1) implies

$$
x^{\prime \alpha} F_{\alpha \beta} \equiv 0,
$$

and it follows that the determinant $\left|F_{\alpha \beta}\right|$ vanishes. We shall make it a part of our hypothesis on $F$ that the rank of this determinant be $n-1$.

For use in examining a certain determinant which will appear presently, we insert here a few miscellaneous observations. As a consequence of equation (3) and the rank of $\left|F_{\alpha \beta}\right|$ the cofactors of the latter satisfy the following relations:

$$
\frac{x^{\prime 1}}{F^{\beta 1}}=\frac{x^{\prime 2}}{F^{\beta 2}}=\cdots=\frac{x^{\prime n}}{F^{\beta n}} .
$$

Noting that the quantities $F^{\alpha \beta}$ are symmetric in their indices, these equalities are seen to be expressible in the form

$$
\frac{x^{\alpha} x^{\beta}}{F^{\alpha \beta}}=\frac{x^{\prime \gamma} x^{\prime \delta}}{F^{\gamma \delta}},
$$

where $\alpha, \beta, \gamma, \delta$ may each be any number of the set $1,2, \cdots, n$ and no summation is to be understood. The reciprocal of the common value of the members of (4) is the $F$ one function of our problem.

The fundamental tensor. It will be recalled that the fundamental covariant tensor of Finsler geometry was constructed by differentiating $\frac{1}{2} F^{2}$ 
with respect to $x^{\prime}$. This may be looked upon as a procedure of differentiating, with respect to the highest derivatives present, a new function $f$ having specific properties which make it equivalent, in some respects, to the original, $F$. The most essential of these properties are the following: (a), the determinant $\left|f_{\alpha \beta}\right|$ must be different from zero (this implies that $f$ be dependent on the parameter); (b), for a certain choice of parameter

$$
\int_{t_{1}}^{t_{2}} f d t=\int_{t_{1}}^{t_{2}} F d t
$$

We adopt the method outlined above and define our new function $f\left(x, x^{\prime}, x^{\prime \prime}\right)$ as follows:

$$
f\left(x, x^{\prime}, x^{\prime \prime}\right)=\left\{H\left(x, x^{\prime}\right)\right\}^{3} F\left(x, x^{\prime}, x^{\prime \prime}\right)+\frac{1}{2}\left\{H^{\prime}\left(x, x^{\prime}\right)\right\}^{2} \cdot{ }^{*}
$$

The function $H$ is to be of class three, homogeneous of degree plus one in $x^{\prime}$ and non-vanishing along all regular curves of our $n$-space. With these restrictions we may so select the parameter that $H$ will maintain the value unity along the curve in question. For such a parameter it is evident that

$$
\int_{t_{1}}^{t_{2}} f\left(x, x^{\prime}, x^{\prime \prime}\right) d t=\int_{t_{1}}^{t_{2}} F\left(x, x^{\prime}, x^{\prime \prime}\right) d t .
$$

Furthermore the determinant of the components of the fundamental tensor

$$
f_{\alpha \beta} \equiv H^{3} F_{\alpha \beta}+H_{\alpha} H_{\beta}
$$

is different from zero along all regular curves, since

$$
\begin{aligned}
\left|f_{\alpha \beta}\right|= & \left|\begin{array}{c}
H_{1} H_{1}+F_{11} \cdots H_{1} H_{n}+F_{1 n} \\
H_{n} H_{1}+F_{n 1} \cdots H_{n} H_{n}+F_{n n}
\end{array}\right|=\left|\begin{array}{ll}
H_{1} H_{1} & F_{12} \cdots F_{1 n} \\
H_{n} H_{1} & F_{n 2} \cdots F_{n n}
\end{array}\right| \\
& +\left|\begin{array}{ll}
F_{11} & H_{1} H_{2} \cdots F_{1 n} \\
F_{n 1} & H_{n} H_{2} \cdots F_{n n}
\end{array}\right|+\cdots+\left|\begin{array}{ll}
F_{11} & F_{12} \cdots F_{1 n} \\
F_{n 1} & F_{n 2} \cdots F_{n n}
\end{array}\right| \\
= & H_{\alpha} H_{\beta} F^{\alpha \beta}=F_{1}^{\prime} x^{\prime \alpha} x^{\prime \beta} H_{\alpha} H_{\beta}=F_{1}^{\prime} \neq 0 .
\end{aligned}
$$

(Here $F_{1}{ }^{\prime}$ represents the $F$ one function, $t$ is the Finsler arc length and $x^{\prime \alpha} H_{\alpha}=H$ since $H$ is homogeneous of degree +1 in $x^{\prime}$.)

Invariants of $F\left(x, x^{\prime}, x^{\prime \prime}\right)$ and $H\left(x, x^{\prime}\right)$. We shall consider $F\left(x, x^{\prime}, x^{\prime \prime}\right)$ as an absolute scalar invariant (that is a tensor of weight and order zero)

* Although we find it convenient to select the parameter in a special way it is desirable to have the quantities $f_{\alpha \beta}$ independent of the parameter and it is for this reason that we introduce the factor $H^{3}$. One readily verifies that $H^{4} F_{\alpha \beta}$ satisfies the set of necessary and sufficient conditions (1), (2) and so we conclude that $H^{3} F_{\alpha \beta}$ has the desired property. Also, we note that $H_{\alpha}$ is homogeneous of degree zero in $x^{\prime}$. 
and accordingly transform it as below by means of the twice extended point transformation

$$
\begin{aligned}
x^{\gamma} & =x^{\gamma}\left(y^{1}, y^{2}, \cdots, y^{n}\right) ; & y^{k} & =y^{k}\left(x^{1}, x^{2}, \cdots, x^{n}\right) ; \\
x^{\prime \gamma} & =\frac{\partial x^{\gamma}}{\partial y^{k}} y^{\prime k} ; & x^{\prime \prime \gamma} & =\frac{\partial^{2} x^{\gamma}}{\partial y^{j} \partial y^{k}} y^{\prime j} y^{\prime k}+\frac{\partial x^{\gamma}}{\partial y^{k}} y^{\prime \prime k} .
\end{aligned}
$$

(We note in passing that the $x^{\prime \gamma}$ are the components of a tensor while the $x^{\prime \prime \gamma}$ are not.) Thus, the law of transformation of $F$ is expressed by the equation.

$$
\bar{F}\left(y, y^{\prime}, y^{\prime \prime}\right)=F\left(x(y), \frac{\partial x}{\partial y^{a}} y^{\prime a}, \frac{\partial x}{\partial y^{a}} y^{\prime \prime a}+\frac{\partial^{2} x}{\partial y^{a} \partial y^{b}} y^{\prime a} y^{\prime b}\right) .
$$

Differentiating this equality with respect to $y^{\prime \prime i}$, we have

$$
\bar{F}_{i}=F_{\alpha} \frac{\partial x^{\alpha}}{\partial y^{i}} .
$$

Hence we see that the $q$ th order partial derivatives $F_{\alpha_{1} \cdots \alpha_{q}}$ of a scalar $F\left(x, x^{\prime}, x^{\prime \prime}\right)$ are the components of a tensor covariant of order $q$, and it is because of this that we have represented these derivatives in a special way. A moment's consideration of the extended point transformation will show that the above statement is true of scalars containing any order derivatives. Differentiating the equation of transformation of $F$, we have

$$
\begin{aligned}
\bar{F}_{y^{\prime i}} & =F_{x^{\prime}} \frac{\partial x^{\alpha}}{\partial y^{i}}+2 F_{\alpha} \frac{\partial^{2} x^{\alpha}}{\partial y^{i} \partial y^{j}} y^{\prime j} \\
\bar{F}_{i}^{\prime} & =F_{\alpha}^{\prime} \frac{\partial x^{\alpha}}{\partial y^{i}}+F_{\alpha} \frac{\partial^{2} x^{\alpha}}{\partial y^{i} \partial y^{j}} y^{\prime j}
\end{aligned}
$$

and we see that

$$
\bar{F}_{y^{\prime i}}-2 \bar{F}_{i}^{\prime}=\left(F_{x^{\prime \alpha}}-2 F_{\alpha}^{\prime}\right) \frac{\partial x^{\alpha}}{\partial y^{i}},
$$

and hence the quantities $\left(F_{x^{\prime \alpha}}-2 F_{\alpha}^{\prime}\right)$ are the components of a covariant tensor. In some respects this tensor is the analogue of $H_{\alpha}$. For if the integral $\int H\left(x, x^{\prime}\right) d t$ is to be independent of the parameter, the following identity must hold:

$$
x^{\prime \alpha} H_{\alpha}=H{ }^{*}
$$

* Oscar Bolza, loc. cit., p. 193. 
The corresponding conditions for $F\left(x, x^{\prime}, x^{\prime \prime}\right)$ are

$$
x^{\prime \alpha} F_{\alpha}=0 ; x^{\prime \alpha} F_{x^{\prime} \alpha}+2 x^{\prime \prime} F_{\alpha}=F,
$$

and since, by the first of these relations, $x^{\prime \prime \alpha} F_{\alpha}=-x^{\prime \alpha} F_{\alpha}^{\prime}$, the last relation may be written in the form

$$
x^{\prime \alpha}\left\{F_{x^{\prime \alpha}}-2 F_{\alpha}^{\prime}\right\}=F .
$$

THEOREM: The left members of the Euler equations associated with the integral

$$
\int_{t_{1}}^{t_{2}} F\left(x, x^{\prime}, x^{\prime \prime}\right) d t
$$

are the components of a covariant tensor of order one.

To prove this we differentiate the equation of transformation of $F$ with respect to $y^{i}$ and the equations of transformation of $\bar{F}_{i}^{\prime}$ and $\bar{F}_{y^{\prime} i}-2 \bar{F}_{i}^{\prime}$, with respect to $t$, and combine; thus

$$
\begin{aligned}
& \bar{F}_{y^{i}}=F_{x^{\alpha}} \frac{\partial x^{\alpha}}{\partial y^{i}}+F_{x^{\prime \alpha}} \frac{\partial^{2} x^{\alpha}}{\partial y^{i} \partial y^{j}} y^{\prime j}+F_{\alpha} \cdot\left(\frac{\partial^{2} x^{\alpha}}{\partial y^{i} \partial y^{j}} y^{\prime \prime}+\frac{\partial^{3} x^{\alpha}}{\partial y^{i} \partial y^{j} \partial y^{k}} y^{\prime} y^{\prime k}\right), \\
& \bar{F}_{i}^{\prime \prime}=F_{\alpha}^{\prime \prime} \frac{\partial x^{\alpha}}{\partial y^{i}}+2 F_{\alpha}^{\prime} \frac{\partial^{2} x^{\alpha}}{\partial y^{i} \partial y^{j}} y^{\prime j}+F_{\alpha} \cdot\left(\frac{\partial^{2} x^{\alpha}}{\partial y^{i} \partial y^{j}} y^{\prime \prime} j+\frac{\partial^{3} x^{\alpha}}{\partial y^{i} \partial y^{j} \partial y^{k}} y^{\prime j} y^{\prime k}\right) \\
&-\left(\bar{F}_{y^{\prime i}}^{\prime}-2 \bar{F}_{i}^{\prime \prime}\right)=-\left(F_{x^{\prime \alpha}}^{\prime}-2 F_{\alpha}^{\prime \prime}\right) \frac{\partial x^{\alpha}}{\partial y^{i}}-\left(F_{x^{\prime \alpha}}-2 F_{\alpha}^{\prime}\right) \frac{\partial^{2} x^{\alpha}}{\partial y^{i} \partial y^{i}} y^{\prime j} \\
& \bar{F}_{y^{i}}-\bar{F}_{y^{\prime i}}^{\prime}+\bar{F}_{i}^{\prime \prime}=\left(F_{x^{\alpha}}-F_{x^{\prime \alpha}}^{\prime}+F_{\alpha}^{\prime \prime}\right) \frac{\partial x^{\alpha}}{\partial y^{i}}
\end{aligned}
$$

It is a theorem of the calculus of variations that this tensor contracted with $x^{\prime}$ is zero.

If $\xi$ and $\eta$ are two vectors defined along a regular curve $C$, we shall call the invariants

$$
\left(\xi^{\alpha} \xi^{\beta} f_{\alpha \beta}\right)^{1 / 2}, \frac{\xi^{\alpha} \eta^{\beta} f_{\alpha \beta}}{\left(\xi^{\alpha} \xi^{\beta} f_{\alpha \beta}\right)^{1 / 2}\left(\eta^{\alpha} \eta^{\beta} f_{\alpha \beta}\right)^{1 / 2}}
$$

(where the arguments of $f_{\alpha \beta}$ are taken along $C$ ) the $\theta$ magnitude of $\xi$ and the $\theta$ cosine of the angle between $\xi$ and $\eta$, with respect to the given curve. Likewise,

$$
\xi^{\alpha} \eta^{\beta} f_{\alpha \beta}
$$

will be referred to as the $\theta$ scalar product of $\xi$ and $\eta$ with respect to $C$. The similar quantities obtained by replacing $f_{\alpha \beta}$ with $I^{2} f_{\alpha \beta}(I \equiv F / H)$ will be 
designated $\tau$ invariants. In all of the subsequent work the arguments of $f$ will be taken along a regular curve and we shall suppose the parameter $t$ to be so chosen that $H\left(x, x^{\prime}\right)$ is unity along the curve in question. As a consequence of this choice of parameter and the homogeneity of $H$, it follows that the tangent vector $x^{\prime}$ of the curve is of $\theta$ magnitude unity, for

$$
x^{\prime \alpha} x^{\beta} f_{\alpha \beta}=1 \text {, }
$$

since

$$
x^{\prime \alpha} F_{\alpha \beta}=0 .
$$

Likewise the $\tau$ magnitude of $d x / d s=x^{\prime} / I \quad\left(s=\int_{t_{1}}^{t} F d t\right)$ is unity.

The differentiation process. The basis of our differentiation process is a relationship analogous to the fundamental affine connection of Riemannian geometry. We form this relationship from the equation of transformation of the first "Christoffel symbol" of our space by replacing irregular terms with quantities having a tensor character.

The law of transformation of the fundamental covariant tensor $f_{\alpha \beta}=H^{3} F_{\alpha \beta}$ $+H_{\alpha} H_{\beta}$ is expressed by the equation

(5) $\bar{f}_{i j}\left(y, y^{\prime}, y^{\prime \prime}\right)=f_{\alpha \beta}\left(x(y), \frac{\partial x}{\partial y^{a}} y^{\prime a}, \frac{\partial x}{\partial y^{a}} y^{\prime \prime a}+\frac{\partial^{2} x}{\partial y^{a} \partial y^{b}} y^{\prime a} y^{\prime b}\right) \frac{\partial x^{\alpha}}{\partial y^{i}} \frac{\partial x^{\beta}}{\partial y^{j}}$.

Differentiating with respect to $y^{k}$ we obtain

$$
\begin{aligned}
\bar{f}_{i j y^{k}}=f_{\alpha \beta} \cdot\left(\frac{\partial^{2} x^{\alpha}}{\partial y^{i} \partial y^{k}} \frac{\partial x^{\beta}}{\partial y^{j}}+\right. & \left.\frac{\partial x^{\alpha}}{\partial y^{i}} \frac{\partial^{2} x^{\beta}}{\partial y^{j} \partial y^{k}}\right) \\
& +\left(f_{\alpha \beta} \gamma \cdot \frac{\partial x^{\gamma}}{\partial y^{k}}+f_{\alpha \beta x^{\prime} \gamma} \cdot \frac{\partial x^{\prime \gamma}}{\partial y^{k}}+f_{\alpha \beta \gamma} \cdot \frac{\partial x^{\prime \prime \gamma}}{\partial y^{k}}\right) \frac{\partial x^{\alpha}}{\partial y^{i}} \frac{\partial x^{\beta}}{\partial y^{k}},
\end{aligned}
$$

from which the Christoffel symbol $[\alpha \beta, \gamma]$ is formed in accordance with the defining equation

$$
[\alpha \beta, \gamma] \equiv \frac{1}{2}\left(f_{\alpha \gamma x \beta}+f_{\gamma \beta x^{\alpha}}-f_{\alpha \beta x^{\gamma}}\right) .
$$

The law of transformation of these symbols is evidently

$$
\begin{aligned}
{[\overline{i j, k}]=} & f_{\alpha \beta} \cdot \frac{\partial^{2} x^{\alpha}}{\partial y^{i} \partial y^{i}} \frac{\partial x^{\beta}}{\partial y^{k}}+[\alpha \beta, \gamma] \frac{\partial x^{\alpha}}{\partial y^{i}} \frac{\partial x^{\beta}}{\partial y^{j}} \frac{\partial x^{\gamma}}{\partial y^{k}} \\
& +\frac{1}{2}\left[\frac{\partial x^{\alpha}}{\partial y^{i}} \frac{\partial x^{\beta}}{\partial y^{k}} \frac{\partial x^{\prime \gamma}}{\partial y^{j}}+\frac{\partial x^{\beta}}{\partial y^{k}} \frac{\partial x^{\alpha}}{\partial y^{j}} \frac{\partial x^{\prime \gamma}}{\partial y^{i}}-\frac{\partial x^{\alpha}}{\partial y^{i}} \frac{\partial x^{\beta}}{\partial y^{j}} \frac{\partial x^{\prime} \gamma}{\partial y^{k}}\right] f_{\alpha \beta x^{\prime} \gamma} \\
& +\frac{1}{2}\left[\frac{\partial x^{\alpha}}{\partial y^{i}} \frac{\partial x^{\beta}}{\partial y^{k}} \frac{\partial x^{\prime \prime \gamma}}{\partial y^{j}}+\frac{\partial x^{\beta}}{\partial y^{k}} \frac{\partial x^{\alpha}}{\partial y^{j}} \frac{\partial x^{\prime \prime \gamma}}{\partial y^{i}}-\frac{\partial x^{\alpha}}{\partial y^{i}} \frac{\partial x^{\beta}}{\partial y^{j}} \frac{\partial x^{\prime \prime \gamma}}{\partial y^{k}}\right] f_{\alpha \beta \gamma} .
\end{aligned}
$$


Multiply both members of (6) by $y^{\prime j}$ and sum. The resulting equation with the lines somewhat rearranged is

$$
\begin{aligned}
{[\overline{i j, k}] y^{\prime j}=} & f_{\alpha \beta} \cdot \frac{\partial^{2} x^{\alpha}}{\partial y^{i} \partial y^{j}} y^{\prime j} \frac{\partial x^{\beta}}{\partial y^{k}}+[\alpha \beta, \gamma] x^{\prime \beta} \frac{\partial x^{\alpha}}{\partial y^{i}} \frac{\partial x^{\gamma}}{\partial y^{k}} \\
& +\frac{1}{2}\left[f_{\alpha \beta \gamma} \cdot \frac{\partial x^{\prime \prime \gamma}}{\partial y^{j}} y^{\prime j}+f_{\alpha \beta x^{\prime} \gamma} \cdot \frac{\partial x^{\prime \gamma}}{\partial y^{j}} y^{\prime j}\right] \frac{\partial x^{\alpha}}{\partial y^{i}} \frac{\partial x^{\beta}}{\partial y^{k}} \\
& +\frac{1}{2}\left[\frac{\partial x^{\beta}}{\partial y^{k}} x^{\prime \alpha} f_{\alpha \beta} x^{\prime} \gamma \cdot \frac{\partial x^{\prime \gamma}}{\partial y^{i}}-\frac{\partial x^{\alpha}}{\partial y^{i}} x^{\prime \beta} f_{\alpha \beta} x^{\prime \gamma} \cdot \frac{\partial x^{\prime \gamma}}{\partial y^{k}}\right. \\
& +\frac{1}{2} x^{\prime \alpha} f_{\alpha \beta \gamma} \cdot \frac{\partial x^{\beta}}{\partial y^{k}} \frac{\partial x^{\prime \prime \gamma}}{\partial y^{i}}-\frac{1}{2} x^{\prime \beta} f_{\alpha \beta \gamma} \cdot \frac{\partial x^{\alpha}}{\partial y^{i}} \frac{\partial x^{\prime \prime \gamma}}{\partial y^{k}}
\end{aligned}
$$

Certain of these terms will be replaced, presently, by equivalent expressions. The last two of the eight terms, however, drop out. In fact we have assumed that $F$ satisfies the identity $x^{\prime \alpha} F_{\alpha}=0$. Differentiating this with respect to $x^{\prime \prime \beta}$ and $x^{\prime \prime \gamma}$, we obtain $x^{\prime \alpha} F_{\alpha \beta \gamma}=0$. But $H\left(x, x^{\prime}\right)$ does not contain $x^{\prime \prime}$ and so $f_{\alpha \beta \gamma}=H^{3} F_{\alpha \beta \gamma}$. Hence (7) reads

$$
\begin{aligned}
{[\overline{i j, k}] y^{\prime j}=} & f_{\alpha \beta} \cdot \frac{\partial^{2} x^{\alpha}}{\partial y^{i} \partial y^{j}} y^{\prime j} \frac{\partial x^{\beta}}{\partial y^{k}}+[\alpha \beta, \gamma] x^{\prime \beta} \frac{\partial x^{\alpha}}{\partial y^{i}} \frac{\partial x^{\gamma}}{\partial y^{k}} \\
& +\frac{1}{2}\left[f_{\alpha \beta \gamma} \cdot \frac{\partial x^{\prime \prime \gamma}}{\partial y^{j}} y^{\prime j}+f_{\alpha \beta x^{\prime} \gamma} \cdot \frac{\partial x^{\prime \gamma}}{\partial y^{j}} y^{\prime j}\right] \frac{\partial x^{\alpha}}{\partial y^{i}} \frac{\partial x^{\beta}}{\partial y^{k}} \\
& +\frac{1}{2}\left[\frac{\partial x^{\beta}}{\partial y^{k}} x^{\prime \alpha} f_{\alpha \beta x^{\prime} \gamma} \cdot \frac{\partial x^{\prime \gamma}}{\partial y^{i}}-\frac{\partial x^{\alpha}}{\partial y^{i}} x^{\prime \beta} f_{\alpha \beta x^{\prime} \gamma} \cdot \frac{\partial x^{\prime \gamma}}{\partial y^{k}}\right]
\end{aligned}
$$

We shall now proceed to develop formulas which are to be used, as just indicated, to modify the form of the right member of (8). The first six formulas listed below are obtained without difficulty from the extended point transformation and consequently require no explanation:

$$
\begin{aligned}
\frac{\partial x^{\prime \gamma}}{\partial y^{j}} & =\frac{\partial^{2} x^{\gamma}}{\partial y^{i} \partial y^{j}} y^{\prime j} \\
x^{\prime \prime \gamma} & =\frac{\partial^{2} x^{\gamma}}{\partial y^{i} \partial y^{j}} y^{\prime i} y^{\prime j}+\frac{\partial x^{\gamma}}{\partial y^{i}} y^{\prime \prime i} ; \\
x^{\prime \prime \prime} \gamma & =\frac{\partial^{3} x^{\gamma}}{\partial y^{i} \partial y^{i} \partial y^{k}} y^{\prime i} y^{\prime j} y^{\prime k}+3 \frac{\partial^{2} x^{\gamma}}{\partial y^{i} \partial y^{j}} y^{\prime \prime i} y^{\prime j}+\frac{\partial x^{\gamma}}{\partial y^{i}} y^{\prime \prime \prime} i \\
y^{\prime \prime j} & =\frac{\partial^{2} y^{j}}{\partial x^{\alpha} \partial x^{\beta}} x^{\prime \alpha} x^{\beta}+\frac{\partial y^{j}}{\partial x^{\beta}} x^{\prime \prime \beta}
\end{aligned}
$$




$$
\frac{\partial x^{\prime \prime \gamma}}{\partial y^{j}}=\left[\frac{\partial^{3} x^{\gamma}}{\partial y^{a} \partial y^{b} \partial y^{j}} y^{\prime a} y^{\prime b}+\frac{\partial^{2} x^{\gamma}}{\partial y^{a} \partial y^{j}} y^{\prime \prime a}\right] y^{\prime j} .
$$

Multiplying (10) by $\partial y^{k} / \partial x^{\gamma}$, summing and changing indices yields

$$
y^{\prime \prime}{ }^{\prime}=-\frac{\partial^{2} x^{\alpha}}{\partial y^{i} \partial y^{k}} y^{\prime i} y^{\prime k} \frac{\partial y^{j}}{\partial x^{\alpha}}+\frac{\partial y^{j}}{\partial x^{\alpha}} x^{\prime \prime \alpha} .
$$

Changing the index $j$ to $k$, in equation (5) differentiating with respect to $y^{\prime j}$, and multiplying by $y^{\prime \prime} j$, we obtain the relation

$$
\bar{f}_{i k y^{\prime j}} \cdot y^{\prime \prime j}=\left[f_{\alpha \beta x^{\prime} \gamma} \cdot \frac{\partial x^{\gamma}}{\partial y^{j}} \cdot y^{\prime \prime j}+2 f_{\alpha \beta \gamma} \cdot \frac{\partial^{2} x^{\gamma}}{\partial y^{a} \partial y^{j}} y^{\prime a} y^{\prime \prime} j\right] \frac{\partial x^{\alpha}}{\partial y^{i}} \frac{\partial x^{\beta}}{\partial y^{j}} \cdot
$$

Multiplying (14) by $f_{\alpha \beta x^{\prime} \gamma} \cdot \partial x^{\gamma} / \partial y^{j}$ and summing $j$, we have

$$
f_{\alpha \beta x^{\prime}} \gamma \cdot \frac{\partial x^{\gamma}}{\partial y^{j}} y^{\prime \prime j}=\left[-\frac{\partial^{2} x^{\gamma}}{\partial y^{i} \partial y^{k}} y^{\prime i} y^{\prime k}+x^{\prime \prime \gamma}\right] f_{\alpha \beta x^{\prime} \gamma} .
$$

Substituting this in (15) and transposing the second term of the right member of (15) we get the relation

$$
\begin{aligned}
-2 f_{\alpha \beta \gamma} \cdot \frac{\partial^{2} x^{\gamma}}{\partial y^{a} \partial y^{j}} \cdot y^{\prime a} y^{\prime \prime} j \cdot \frac{\partial x^{\alpha}}{\partial y^{i}} \frac{\partial x^{\beta}}{\partial y^{k}}=\left[-\frac{\partial^{2} x^{\gamma}}{\partial y^{a} \partial y^{j}} y^{\prime a} y^{\prime j}+x^{\prime \prime \gamma}\right] f_{\alpha \beta x^{\prime} \gamma} \\
\cdot \frac{\partial x^{\alpha}}{\partial y^{i}} \frac{\partial x^{\beta}}{\partial y^{k}}-\bar{f}_{i k y^{\prime j} \cdot y^{\prime \prime} i} .
\end{aligned}
$$

The addition of the identity

$$
2 \frac{\partial^{2} x^{\gamma}}{\partial y^{a} \partial y^{j}} y^{\prime \prime a} y^{\prime}-2 \frac{\partial^{2} x^{\gamma}}{\partial y^{a} \partial y^{j}} y^{\prime \prime a} y^{\prime j}=0
$$

to (13) and the multiplication of the result by $\frac{1}{2} f_{\alpha \beta \gamma}\left(\partial x^{\alpha} / \partial y^{i}\right)\left(\partial x^{\beta} / \partial y^{k}\right)$ yields the equality

$$
\begin{aligned}
\frac{1}{2}\left\{f_{\alpha \beta \gamma} \cdot \frac{\partial x^{\prime \prime \gamma}}{\partial y^{j}} y^{\prime j}=\right. & f_{\alpha \beta \gamma}\left[\frac{\partial^{3} x^{\gamma}}{\partial y^{a} \partial y^{b} \partial y^{j}} y^{\prime a} y^{\prime b} y^{j}+3 \frac{\partial^{2} x^{\gamma}}{\partial y^{a} \partial y^{j}} y^{\prime \prime a} y^{\prime j}\right] \\
& \left.-2 f_{\alpha \beta \gamma} \frac{\partial^{2} x^{\gamma}}{\partial y^{a} \partial y^{j}} y^{\prime \prime a} y^{\prime j}\right\} \frac{\partial x^{\alpha}}{\partial y^{i}} \frac{\partial x^{\beta}}{\partial y^{k}} .
\end{aligned}
$$

By adding

$$
\left\{\frac{1}{2} f_{\alpha \beta x^{\prime}} \frac{\partial x^{\prime \gamma}}{\partial y^{j}} y^{\prime j}=\frac{1}{2} f_{\alpha \beta x^{\prime} \gamma} \frac{\partial x^{\prime \gamma}}{\partial y^{j}} y^{\prime j}\right\} \frac{\partial x^{\alpha}}{\partial y^{i}} \frac{\partial x^{\beta}}{\partial y^{k}}
$$


and substituting by means of (11) and (16), the relation (17) may be put into the symmetric form

$$
\begin{gathered}
{\left[\frac{1}{2} f_{\alpha \beta \gamma} \frac{\partial x^{\prime \prime \gamma}}{\partial y^{j}} y^{\prime j}+\frac{1}{2} f_{\alpha \beta x^{\prime} \gamma} \frac{\partial x^{\prime \gamma}}{\partial y^{j}} y^{\prime j}\right] \frac{\partial x^{\alpha}}{\partial y^{i}} \frac{\partial x^{\beta}}{\partial y^{k}}} \\
=\frac{1}{2} f_{\alpha \beta \gamma}\left[x^{\prime \prime \prime \gamma}-y^{\prime \prime \prime} a \frac{\partial x^{\gamma}}{\partial y^{a}}\right] \frac{\partial x^{\alpha}}{\partial y^{i}} \frac{\partial x^{\beta}}{\partial y^{k}}+\frac{1}{2}\left[-\frac{\partial^{2} x^{\gamma}}{\partial y^{a} \partial y^{\prime}} y^{\prime a} y^{\prime j}+x^{\prime \prime \gamma}\right] f_{\alpha \beta x^{\prime} \gamma} \\
\cdot \frac{\partial x^{a}}{\partial y^{i}} \frac{\partial x^{\beta}}{\partial y^{k}}-\frac{1}{2} \bar{f}_{i k y^{\prime} j} \cdot y^{\prime \prime j}+\frac{1}{2} f_{\alpha \beta x^{\prime} \gamma} \frac{\partial x^{\prime \gamma}}{\partial y^{j}} y^{\prime j} \frac{\partial x^{\alpha}}{\partial y^{i}} \frac{\partial x^{\beta}}{\partial y^{k}}
\end{gathered}
$$

Since

$$
\frac{\partial x^{\prime \gamma}}{\partial y^{j}} y^{\prime j}=\frac{\partial^{2} x^{\gamma}}{\partial y^{a} \partial y^{j}} y^{\prime a} y^{\prime j}
$$

the third and sixth terms of the right member of (18) cancel. Differentiating the identity $x^{\prime \beta} F_{\beta}=0$ we see that $x^{\prime \beta} F_{\alpha \beta x^{\prime} \gamma}=-F_{\alpha \gamma}$ and so $x^{\prime \beta} f_{\alpha \beta x^{\prime} \gamma}=x^{\prime \beta}\left[H^{3} F_{\alpha \beta x^{\prime} \gamma}\right.$ $\left.+3 H^{2} H_{\gamma} F_{\alpha \beta}+H_{\alpha \gamma} H_{\beta}+H_{\alpha} H_{\beta \gamma}\right]=-H^{3} F_{\alpha \gamma}+H H_{\alpha \gamma}$, that is, $x^{\prime \beta} f_{\alpha \beta x^{\prime} \gamma}$ is a symmetric covariant tensor.

We now express $\partial x^{\prime} \gamma / \partial y^{i}$ by means of Taylor's differentiation scheme, $\dagger$ designating the expression $\frac{1}{2} H^{2}$ by means of $h$; thus

where

$$
\frac{\partial x^{\prime \gamma}}{\partial y^{i}}=\bar{T}_{i}{ }^{m} \frac{\partial x^{\gamma}}{\partial y^{m}}-T_{\alpha^{\gamma}} \frac{\partial x^{\alpha}}{\partial y^{i}},
$$

$$
\begin{aligned}
& \bar{T}_{i}^{m}=\bar{\Gamma}_{i j}^{* m} y^{\prime j}+\frac{1}{2} y^{\prime \prime k} \bar{h}_{i j k} \bar{h}^{j m} \\
& T_{\alpha}^{\gamma}=\Gamma_{\alpha \beta}^{* \gamma} x^{\prime \beta}+\frac{1}{2} x^{\prime \prime \delta} h_{\alpha \beta \delta} h^{\beta \gamma}
\end{aligned}
$$

(the $\Gamma^{*}$ being the Christoffel symbols of the second kind associated with the Finsler space $\left.H\left(x, x^{\prime}\right)\right)$.

Multiplying the above equation for $\partial x^{\prime \gamma} / \partial y^{i}$ by $\left(\partial x^{\alpha} / \partial y^{k}\right)\left(x^{\beta \beta} f_{\alpha \beta x^{\prime}}\right)$ we get

$$
\begin{aligned}
\frac{\partial x^{\alpha}}{\partial y^{k}} x^{\prime \beta} f_{\alpha \beta x^{\prime} \gamma} \frac{\partial x^{\prime \gamma}}{\partial y^{i}} & =\frac{\partial x^{\alpha}}{\partial y^{k}} x^{\prime \beta} f_{\alpha \beta x^{\prime}}\left[\bar{T}_{i}{ }^{m} \frac{\partial x^{\gamma}}{\partial y^{m}}-T_{\alpha^{\gamma}} \frac{\partial x^{\alpha}}{\partial y^{i}}\right] \\
& =y^{\prime} \bar{f}_{k j y^{\prime}} \bar{T}_{i}^{m}-x^{\prime \beta} f_{\gamma \beta x^{\prime \lambda}} T_{\alpha^{\lambda}} \frac{\partial x^{\alpha}}{\partial y^{i}} \frac{\partial x^{\gamma}}{\partial y^{k}} .
\end{aligned}
$$

$\dagger$ J. H. Taylor, A generalization of Levi-Civita's parallelism and the Frenet fomulas, loc. cit., p. 255 , formula 21 . 
Equations (18) and (19) will now be used to modify the form of equation (8). In fact we modify the second line of (8) by means of (18) and the third line by means of (19), thus

$$
\begin{aligned}
{[\overline{i j, k}] y^{\prime j} } & +\frac{1}{2} \bar{f}_{i k y^{\prime j}} y^{\prime \prime}{ }^{i}+\frac{1}{2} \bar{f}_{i k j} y^{\prime \prime \prime} j-\frac{1}{2} y^{\prime} \bar{f}_{k i y^{\prime m}} \bar{T}_{i}^{m} \\
& +\frac{1}{2} y^{\prime} \bar{f}_{i j y^{\prime \prime}} \bar{T}_{k}^{m}=f_{\alpha \beta} \frac{\partial x^{\beta}}{\partial y^{k}} \frac{\partial^{2} x^{\alpha}}{\partial y^{i} \partial y^{j}} y^{\prime j}+\left[[\alpha \beta, \gamma] x^{\prime \beta}\right. \\
& +\frac{1}{2} f_{\alpha \gamma x^{\prime \lambda}} x^{\prime \prime \lambda}+\frac{1}{2} f_{\alpha \gamma \lambda} x^{\prime \prime \prime \prime}-\frac{1}{2} x^{\prime \beta} f_{\gamma \beta x^{\prime \prime}} T_{\alpha^{\lambda}} \\
& \left.+\frac{1}{2} x^{\prime \beta} f_{\alpha \beta x^{\prime \lambda}} T_{\gamma}^{\lambda}\right] \frac{\partial x^{\alpha}}{\partial y^{i}} \frac{\partial x^{\gamma}}{\partial y^{k}} .
\end{aligned}
$$

We shall write this last equation symbolically as

$$
[\overline{i, k}]=f_{\alpha \beta} \frac{\partial x^{\beta}}{\partial y^{k}} \frac{\partial^{2} x^{\alpha}}{\partial y^{i} \partial y^{j}} y^{\prime i}+[\alpha, \gamma] \frac{\partial x^{\alpha}}{\partial y^{i}} \frac{\partial x^{\gamma}}{\partial y^{k}} .
$$

Multiplying both sides of this equation by $\partial y^{k} / \partial x^{\rho}$, we obtain

$$
[\overline{i, k}] \frac{\partial y^{k}}{\partial x^{\rho}}=f_{\alpha \rho} \frac{\partial^{2} x^{\alpha}}{\partial y^{i} \partial y^{j}} y^{j}+[\alpha, \rho] \frac{\partial x^{\alpha}}{\partial y^{i}} .
$$

Multiply by fop and note

$$
f^{\sigma \rho} \frac{\partial y^{k}}{\partial x^{\rho}}=\bar{f}^{l k} \frac{\partial x^{\sigma}}{\partial v^{l}} .
$$

The result is

$$
\bar{f}^{l k} \frac{\partial x^{\sigma}}{\partial y^{l}}[\bar{i}, \bar{k}]=\frac{\partial^{2} x^{\sigma}}{\partial y^{i} \partial y^{j}} y^{\prime i}+[\alpha, \rho] f^{\sigma \rho} \frac{\partial x^{\alpha}}{\partial y^{i}} .
$$

Application to covariant vectors. Differentiating the equation of transformation of a covariant vector $V$,

$$
\bar{V}_{i}=V_{\sigma} \frac{\partial x^{\sigma}}{\partial y^{i}}
$$

with respect to $t$, multiplying $\left(20^{\prime}\right)$ by $-V^{\sigma}$, and adding, we obtain

$$
\bar{V}_{i}^{\prime}-\bar{V}_{l} \bar{f}^{l k}\left[\overline{i, k]}=\left\{V_{\sigma}^{\prime}-V_{\delta} f^{\delta \rho}[\sigma, \rho]\right\} \frac{\partial x^{\sigma}}{\partial y^{i}} .\right.
$$

Indicating this operation on $V_{i}$ by $\theta V_{i}$ we have as a conclusion 
If $V_{\sigma}$ is a covariant tensor of rank one, then $\theta V_{\sigma}$ is also a covariant tensor of rank one.

A similar process may be applied to contravariant vectors. For, if $V^{*}$ is a contravariant vector, then

and

$$
V^{\sigma}=\bar{V}^{i} \frac{\partial x^{\sigma}}{\partial y^{i}}
$$

$$
V^{\prime \sigma}=\bar{V}^{\prime i} \frac{\partial x^{\alpha}}{\partial y^{i}}+\bar{V}^{i} \frac{\partial^{2} x^{\sigma}}{\partial y^{i} \partial y^{j}} y^{\prime j} .
$$

Eliminating the second derivative as before gives the result

$$
\begin{aligned}
V^{\prime \sigma}+V^{\alpha}[\alpha, \rho] f^{\sigma \rho} & =\bar{V}^{\prime i} \frac{\partial x^{\sigma}}{\partial y^{i}}+\bar{V}^{i}[\overline{i, k}] \bar{f}^{\prime k} \frac{\partial x^{\sigma}}{\partial y^{l}} \\
& =\left[\bar{V}^{\prime i}+\bar{V}^{i}[\overline{j, k}] \bar{f}^{i k}\right] \frac{\partial x^{\sigma}}{\partial y^{i}} .
\end{aligned}
$$

Designating this process as before by $\theta$ we have

If $V^{\sigma}$ is a contravariant vector, then $\theta V^{\sigma}$ is a contravariant vector.

In general the $\theta$ process, with respect to a given curve, will consist of forming the derivative of the tensor $X:::$, with respect to $t$, and adding

$$
X^{\alpha}:::[\alpha, \rho] f^{\sigma \rho}
$$

for each contravariant index, and subtracting

$$
X_{\delta}::: f^{\delta} \rho[\sigma, \rho]
$$

for each covariant index, all arguments being taken along the curve in question.

We next point out that the rule of ordinary calculus for forming the derivative of a product is conserved. It will suffice for this to examine a special case, say that of the product $\bar{V}_{i} \bar{V}_{k}$. By the law of compusition of tensors $\bar{V}_{i} \bar{V}_{k}$ is a second-order covariant tensor and so

Then

$$
\bar{V}_{i} \bar{V}_{k}=V_{\sigma} V_{\tau} \frac{\partial x^{\sigma}}{\partial y^{i}} \frac{\partial x^{r}}{\partial y^{k}} .
$$

$$
\begin{aligned}
\bar{V}_{i}^{\prime} \bar{V}_{k}+\bar{V}_{i} \bar{V}_{k}^{\prime}=\left(V_{\sigma}^{\prime} V_{\tau}+V_{\sigma} V_{\tau}^{\prime}\right) & \frac{\partial x^{\sigma}}{\partial y^{i}} \frac{\partial x^{\tau}}{\partial y^{k}} \\
& +V_{\sigma} V_{\tau}\left[\frac{\partial x^{\sigma}}{\partial y^{i}} \frac{\partial^{2} x^{\tau}}{\partial y^{k} \partial y^{j}} y^{\prime i}+\frac{\partial^{2} x^{\sigma}}{\partial y^{i} \partial y^{j}} y^{\prime j} \frac{\partial x^{\tau}}{\partial y^{k}}\right] .
\end{aligned}
$$


Hence

$$
\begin{aligned}
\bar{V}_{i}^{\prime} \bar{V}_{k}+\bar{V}_{i} \bar{V}_{k}^{\prime}= & \left(V_{\sigma}^{\prime} V_{\tau}+V_{\sigma} V_{\tau}^{\prime}\right) \frac{\partial x^{\sigma}}{\partial y^{i}} \frac{\partial x^{\tau}}{\partial y^{k}} \\
& +V_{\sigma} V_{r} \frac{\partial x^{\sigma}}{\partial y^{i}}\left[\bar{f}^{l m} \frac{\partial x^{\tau}}{\partial y^{l}}\left[\overline{k, m}-[\alpha, \rho] f^{\tau \rho} \frac{\partial x^{\alpha}}{\partial y^{k}}\right]\right. \\
& +V_{\sigma} V_{\tau} \frac{\partial x^{\tau}}{\partial y^{k}}\left[\bar{f}^{l m} \frac{\partial x^{\sigma}}{\partial y^{l}}\left[\overline{i, m}-[\alpha, \rho] f^{\sigma \rho} \frac{\partial x^{\alpha}}{\partial y^{i}}\right]\right.
\end{aligned}
$$

Transposing, we get

$$
\begin{aligned}
\bar{V}_{i}^{\prime} \bar{V}_{k}+\bar{V}_{i} \bar{V}_{k}^{\prime}- & \bar{V}_{i} \bar{V}_{l} \bar{f}^{l m}[\overline{k, m}]-\bar{V}_{l} \bar{V}_{k} \bar{f}^{l m}[\overline{i, m}] \\
& =\left[V_{\sigma}^{\prime} V_{\tau}+V_{\sigma} V_{\tau}^{\prime}-V_{\sigma} V_{\alpha} f^{\alpha \rho}[\tau, \rho]-V_{\alpha} V_{\tau} f^{\alpha \rho}[\sigma, \rho]\right] \frac{\partial x^{\sigma}}{\partial y^{i}} \frac{\partial x^{\tau}}{\partial y^{k}}
\end{aligned}
$$

Rewriting the above we have, for the left member,

$$
\left[\bar{V}_{i}^{\prime}-\bar{V}_{l} \bar{f}^{l m}[\overline{i, m}]\right] \bar{V}_{k}+\bar{V}_{i}\left[\bar{V}_{k}^{\prime}-\bar{V}_{l} \bar{f}^{l m}[\overline{k, m}]\right]
$$

that is,

$$
\theta\left(\bar{V}_{i} \bar{V}_{k}\right)=\left(\theta \bar{V}_{i}\right) \bar{V}_{k}+\bar{V}_{i}\left(\theta \bar{V}_{k}\right)
$$

The $\theta$ process applied to a scalar expressed as the contraction of tensors is equivalent to the ordinary derivative.

Let us consider, for example, the scalar $V_{\sigma} V^{\sigma}$. Forming the $\theta$ derivative of this by the rule just discussed, we have

$$
\theta\left(V_{\sigma} V^{\sigma}\right)=\left(\theta V_{\sigma}\right) V^{\sigma}+V_{\sigma}\left(\theta V^{\sigma}\right)
$$

that is,

$$
\begin{aligned}
\theta\left(V_{\sigma} V^{\sigma}\right) & =\left[V_{\sigma}^{\prime}-V_{\delta} f^{\delta^{\rho}}[\sigma, \rho]\right] V^{\sigma}+V_{\sigma}\left[V^{\prime \sigma}+V^{\alpha}[\alpha, \rho] f^{\sigma \rho}\right] \\
& =V^{\prime} V_{\sigma}+V_{\sigma}^{\prime} V^{\sigma} .
\end{aligned}
$$

A most important property of our $\theta$ process is expressed by the equation

$$
\theta f_{\alpha \gamma} \equiv 0 \text {. }
$$

We have

$$
\begin{aligned}
\theta f_{\alpha \gamma} & =f_{\alpha \gamma}^{\prime}-f_{\sigma \gamma} f^{\sigma \rho}[\alpha, \rho]-f_{\alpha \sigma} f^{\sigma \rho}[\gamma, \rho] \\
& =f_{\alpha \gamma}^{\prime}-[\alpha, \gamma]-[\gamma, \alpha] .
\end{aligned}
$$

One readily verifies that




Then, since

it follows that

$$
[\alpha \beta, \gamma]+[\gamma \beta, \alpha]=\frac{\partial f_{\alpha \gamma}}{\partial x^{\beta}}
$$

$$
\theta f_{\alpha \gamma} \equiv 0 \text {. }
$$

If a contravariant vector $\xi^{\alpha}$, defined at each point of a curve, satisfies the differential equations

$$
\theta \xi^{\alpha}=0 \quad(\alpha=1,2, \cdots, n)
$$

along the curve, we shall say the vector remains parallel $\theta$. This parallelism reduces to Taylor's if $F_{\alpha \beta}$ and $F$ are functions of $x$ and $x^{\prime}$ alone and $H$ is chosen as $F$.

THEOREM. If two vectors, $\xi$ and $\eta$, defined along a curve, each remain parallel $\theta$ al.ong the curve, then the $\theta$ magnitude of the vectors and the $\theta$ cosine of the angle between them are constant along the curve.

Each of the three expressions

$$
f_{\alpha \beta} \xi^{\alpha} \xi^{\beta}, \quad f_{\alpha \beta} \eta^{\alpha} \eta^{\beta}, \quad f_{\alpha \beta} \xi^{\alpha} \eta^{\beta}
$$

is a scalar formed by the contraction of tensors, and so the $\theta$ process applied to them is equivalent to differentiating them in the ordinary way with respect to $t$. Thus, we have

and

$$
\frac{d}{d t}\left[f_{\alpha \beta} \xi^{\alpha} \xi^{\beta}\right]=\theta\left[f_{\alpha \beta} \xi^{\alpha} \xi^{\beta}\right]=\left[\theta f_{\alpha \beta}\right] \xi^{\alpha} \xi^{\beta}+2 f_{\alpha \beta}\left(\theta \xi^{\alpha}\right) \xi^{\beta}=0,
$$

$$
\frac{d}{d t}\left[f_{\alpha \beta} \xi^{\alpha} \eta^{\beta}\right]=\left[\theta f_{\alpha \beta}\right] \xi^{\alpha} \eta^{\beta}+f_{\alpha \beta}\left(\theta \xi^{\alpha}\right) \eta^{\beta}+f_{\alpha \beta} \xi^{\alpha}\left(\theta \eta^{\beta}\right)=0
$$

since $\theta f_{\alpha \beta}=0$, and $\theta \xi^{\alpha}=\theta \eta^{\beta}=0$ by hypothesis. And so the two cardinal properties of parallelism are preserved.

Of special interest, perhaps, are the curves along which the fundamental tensors $f_{\alpha \beta}$ and $h_{\alpha \beta}\left(h_{\alpha \beta}=H H_{\alpha \beta}+H_{\alpha} H_{\beta}\right)$ coincide. Let $C$ be one of these curves and let us apply the $\theta$ process to a vector $V^{\sigma}$ defined along $C$; thus

$$
\begin{aligned}
\theta V^{\sigma}= & V^{\prime \sigma}+V^{\alpha}[\alpha, \rho] f^{\sigma \rho} \\
= & V^{\prime \sigma}+V^{\alpha}\left\{[\alpha \beta, \rho] x^{\prime \beta}+\frac{1}{2} f_{\alpha \rho x} \lambda \cdot x^{\prime \prime \lambda}+\frac{1}{2} f_{\alpha \rho \lambda} \cdot x^{\prime \prime \prime \lambda}\right. \\
& -\frac{1}{2} x^{\prime \beta} f_{\rho \beta x^{\prime \lambda}} \cdot T_{\alpha}^{\lambda}+\frac{1}{2} x^{\prime \beta} f_{\left.\alpha \beta x^{\lambda} \lambda T_{\rho}^{\lambda}\right\} f^{\sigma \rho} .}
\end{aligned}
$$

We have noted that $x^{\beta} f_{\rho \beta x} \lambda=-H^{3} F_{\rho \lambda}+H H_{\rho \lambda}$, and so the last two terms of $\theta V$ reduce to zero. A moment's consideration will be sufficient to verify that 


$$
\begin{aligned}
x^{\beta}[\alpha \beta, \rho] & =x^{\beta}[\alpha \beta, \rho]^{\prime}-\frac{1}{2} \frac{\partial}{\partial x^{\beta}}\left(H H_{\alpha \rho}\right) \cdot x^{\prime \beta}+\frac{1}{2} \frac{\partial}{\partial x^{\beta}}\left(H^{3} F_{\alpha \rho}\right) \cdot x^{\prime \beta} \\
& =x^{\beta}[\alpha \beta, \rho]^{\prime}-\frac{1}{2} h_{\alpha \rho x^{\beta}} \cdot x^{\beta}+f_{\alpha \rho x \beta} \cdot x^{\prime \beta} .
\end{aligned}
$$

(The symbol $[\alpha \beta, \rho]^{\prime}$ appearing above is used to designate the first Christoffel symbol of the Finsler space $H\left(x, x^{\prime}\right)$.) Moreover

$$
f_{\alpha \rho}^{\prime}-h_{\alpha \rho x \beta} \cdot x^{\prime \beta}=h_{\alpha \beta \beta} \cdot x^{\prime \prime \beta}
$$

since $h_{\alpha \beta}=f_{\alpha \beta}$. Hence we conclude that, with respect to $C$, the Taylor-Synge derivatives* of $V^{\sigma}$ and $\theta V^{\sigma}$ are identical. This property is somewhat analogous to the defining property of Levi-Civita's parallelism, namely: A vector $W$ defined along a curve $K$ of the surface $S$ is said to remain parallel provided it remains parallel when $K$ is considered as belonging to the developable surface tangent to $S$ along $K$.

A second differentiation process, which we shall call the $\tau$ process, may be obtained by multiplying (20) by $I^{2}$ and adding $I I^{\prime}$ to both members. Evidently $\tau\left(I^{2} f_{\alpha \beta}\right)=0$ and consequently the " $\tau$ magnitude" and the " $\tau$ direction" of vectors undergoing a displacement parallel $(\tau)$ are unaltered.

$$
\begin{gathered}
I=\left[1+\frac{1}{2} \frac{\left(x^{\prime} y^{\prime \prime}-y^{\prime} x^{\prime \prime}\right)^{2}}{\left(x^{\prime 2}+y^{\prime 2}\right)^{3}}\right] ; \quad H=\left(x^{\prime 2}+y^{\prime 2}\right)^{1 / 2} \\
F=I H ; \frac{\partial F}{\partial x^{\prime \prime}}=-\frac{\left(x^{\prime} y^{\prime \prime}-y^{\prime} x^{\prime \prime}\right) y^{\prime}}{\left(x^{\prime 2}+y^{\prime 2}\right)^{5 / 2}} ; \frac{\partial F}{\partial y^{\prime \prime}}=\frac{\left(x^{\prime} y^{\prime \prime}-y^{\prime} x^{\prime \prime}\right) x^{\prime}}{\left(x^{\prime 2}+y^{\prime 2}\right)^{5 / 2}} \\
\frac{\partial^{2} F}{\partial x^{\prime \prime} \partial x^{\prime \prime}}=\frac{y^{\prime 2}}{\left(x^{\prime 2}+y^{\prime 2}\right)^{5 / 2}} ; \frac{\partial^{\prime} F}{\partial x^{\prime \prime} \partial y^{\prime \prime}}=\frac{-x^{\prime} y^{\prime}}{\left(x^{\prime 2}+y^{\prime 2}\right)^{5 / 2}} ; \frac{\partial^{2} F}{\partial y^{\prime \prime} \partial y^{\prime \prime}}=\frac{x^{\prime 2}}{\left(x^{\prime 2}+y^{\prime 2}\right)^{5 / 2}} \\
\frac{\partial^{2} H}{\partial x^{\prime} \partial x^{\prime}}=\frac{y^{\prime 2}}{\left(x^{\prime 2}+y^{\prime 2}\right)^{3 / 2}} ; \frac{\partial^{2} H}{\partial x^{\prime} \partial y^{\prime}}=\frac{-x^{\prime}}{\left(x^{\prime 2}+y^{\prime 2}\right)^{3 / 2}} ; \frac{\partial^{2} H}{\partial y^{\prime} \partial y^{\prime}}=\frac{x^{\prime 2}}{\left(x^{\prime 2}+y^{\prime 2}\right)^{3 / 2}}
\end{gathered}
$$

We note that $I$ is independent of the parameter, the determinant $F_{\alpha \beta}$ is of rank $n-1$, the $F$ one function is not zero, and $f_{\alpha \beta}=h_{\alpha \beta}\left(f_{\alpha \beta}=H^{3} F_{\alpha \beta}+H_{\alpha} H_{\beta}\right.$, $\left.h_{\alpha \beta}=H H_{\alpha \beta}+H_{\alpha} H_{\beta}\right)$ : The geometry of this space is very similar to euclidean geometry. The measurement of angles, magnitude of vectors and parallel displacement of vectors are the same as in the euclidean plane. In particular the autoparallel curves of both spaces are straight lines and the two metrics $F$ and $H$ coincide along straight lines.

* See J. H. Taylor, loc. cit., p. 255.

UnIVERSITY OF TEXas, Austin, Texas 